\title{
Histopathological alteration in organs of adult male frog after exposure to alpha- cypermethrin
}

\author{
Ayesha Riaz ${ }^{1^{*}}$, Marium Majeed ${ }^{1}$, Muhammad Ahsan Riaz ${ }^{2}$, Asia Iqbal ${ }^{3}$ \\ and Usman Ali Ashfaq ${ }^{4}$ \\ 1. Department of Zoology, GC Women University Faisalabad, Pakistan \\ 2. Department of Environmental Sciences and Engineering, GC University Faisalabad, Pakistan \\ 3. Department of Wildlife and Ecology, University of Veterinary and Animal Sciences. Lahore, Pakistan \\ 4. Department of Bioinformatics and Biotechnology, GC University Faisalabad, Pakistan \\ *Corresponding author's email: dr.ayeshariaz@gcwuf.edu.pk
}

Citation

Ayesha Riaz, Marium Majeed, Muhammad Ahsan Riaz, Asia Iqbal and Usman Ali Ashfaq. Histopathological alteration in organs of adult male frog after exposure to alpha-cypermethrin. Pure and Applied Biology. Vol. 11, Issue 1, pp175-180. http://dx.doi.org/10.19045/bspab.2022.110019

\begin{tabular}{llll}
\hline \hline Received: $27 / 02 / 2021$ & Revised: 23/04/2021 & Accepted: 02/05/2021 & Online First: 21/05/2021 \\
\hline
\end{tabular}

\section{Abstract}

Alpha-cypermethrin (CYP) is used as an insecticide and indoor residual spray (IRS) in all central towns of Punjab Pakistan with end products sink in water bodies. Habitat loss due to high exposure to insecticides is one of primary factors contributing to amphibian population decline. The extensive industrial and domestic uses of CYP promote many questions about their undesirable health effects. Due to its lipophilic nature CYP affects the immune, cardiovascular, nervous and reproductive systems of organisms in addition to inducing mutagenicity and carcinogenicity with adverse health effects. Current study is designed to investigate the histopathological effect of CYP on target organs of frog as biomarkers of toxicity in frog organs. The frogs were experimentally exposed to sub-lethal concentration $(7.5 \mu \mathrm{g} / \mathrm{L})$ of Alpha-cypermethrin $10 \%$ SC for 14 days. Present study demonstrates health effects that have been associated with exposure to alpha-cypermethrin in terms of damages in kidney, spleen, heart and tongue of frog.

Keywords: Alpha-cypermethrin; Frog; Histopathology; Insecticides; Pyrethroid; Toxicity

\section{Introduction}

Cypermethrin (CYP) is widely used by farmers around the world, especially in developing countries as leading insecticide for agricultural and public health purposes [1]. CYP toxic affects cause abnormal reactive oxygen species (ROS) production and substantial damage to the structure of cells, carbohydrates, nucleic acids, lipids and protein [2].

Cypermethrin, the 2, 2-dimethyl-3cyclopropane-carboxylic acid alpha-cyano- 3-phenoxybenzyl ester, is a synthetic pyrethroid, similar to natural pyrethrins, so far more environmentally safe. The structure is based on chrysanthemum pyrethrum, an extract from dried flowers used as a nonsystemic pesticide that works through touch and ingestion to control a wide variety of cotton, cereal, vegetable and fruit crops related insects. Pyrethroid pesticides generate toxic effects on human health and biota in general, are considered a question of 
continuous study and evaluation at global level [3].

Through soil, air, water, and even in animal and human tissues poisonous chemicals such as pesticides have been reported causing injurious effects and even deaths [4]. Several studies have shown that cypermethrin induces oxidative stress to destroy the brain, liver, sperm, and erythrocytes [5].

In the context of amphibians' population decline in agricultural regions, pesticide pollution is considered to be a significant factor [6]. Pyrethroid insecticides are synthetic molecules that are structurally linked to natural pyrethrins and share many features to DDT [7]. Amphibian can survive in modified landscape, but careful assessment of long-term resolution is required. The primary factors contributing to amphibian decline is habitat loss due to excessive use of pesticides and insecticides. Alpha-Cypermethrin a lipophilic compound is used as indoor residual spray (IRS) in Punjab Pakistan for the control of dengue mosquitoes. Due to toxic effect on amphibians, these lipophilic compounds are characterized as damaging agents [8].

Studies on animals have observed increased liver weight, increased liver enzymes, hepatocellular hypertrophy, stuffiness and other microscopic changes in organs [9].

The current study is designed to evaluate degradation and lethal effects of alphacypermethrin after acute sub-chronic exposure in male frog by studying the histopathological effects on kidney, spleen, heart and tongue.

\section{Materials and Methods}

For the current study 12 adult male frogs with average weight $( \pm 100 \mathrm{~g})$ were kept in water tanks with standardized environmental conditions. Frogs were randomly divided into two groups; One control group $(n=6)$, and other group with acute sub-chronic exposure $(n=6)$. Two replicates were tested for treated and controls were set up simultaneously with dechlorinated water without administration of alphacypermethrin.

Frogs acclimatization was done in glass containers $(20 \mathrm{~L})$ filled with tap water for 14 days prior to the experiments. Containers were kept in slant to provide the option of both aqueous and dry environment conditions. Frogs were fed with earthworms twice daily. The experimental animals were collected from Asia Scientific trades Jinnah colony Faisalabad.

\section{Test chemical used}

RUBI ${ }^{\circledR} 100$ SC. $\alpha$-cypermethrin analytical standard (applicant: tagros chemicals India Pvt. Ltd, Active Ingredient: $\alpha$-cypermethrin, AI Concentration: 10\%, Formulation Type: $\mathrm{SC}$ ) in quantity $7.5 \mu \mathrm{g} / \mathrm{L}$ mixed with dechlorinated tap water (WHO, 2017).

\section{Organ procurement and histopathological} analysis

After sacrificing frogs, kidney, spleen, heart, and tongue were excised out and fixed using formalin (Sigma Aldrich, USA). For processing, tissues were treated with $70 \%$, $80 \%, 95 \%$, and $100 \%$ ethanol respectively. Finally, the tissues were treated with xylene and embedded in paraffin for making sections with thickness at $5-\mu \mathrm{m}$ for Microtomy (Microm, Germany). The Hematoxylin and eosin staining carried out to assess the morphological variations [10].

\section{Results}

In current study histopathological alterations in different organs (kidney, spleen, heart and tongue) of frog following exposure to CYP were examined using a light microscope. Results of current study revealed recognizable changes in kidney tissues of CYP exposed group. CYP induced morphological changes in the treated group kidney (Fig. 1). At the level of kidney tubules, alpha-cypermethrin induced histological changes such as impairment of kidney, vacuolization, renal tubular degeneration and enlarged capillaries (Fig. 
1a). On the other hand, the tissue study of the kidney after $\mathrm{H}$ and $\mathrm{E}$ staining of the control frog exhibited normal structure (Fig. 1b).
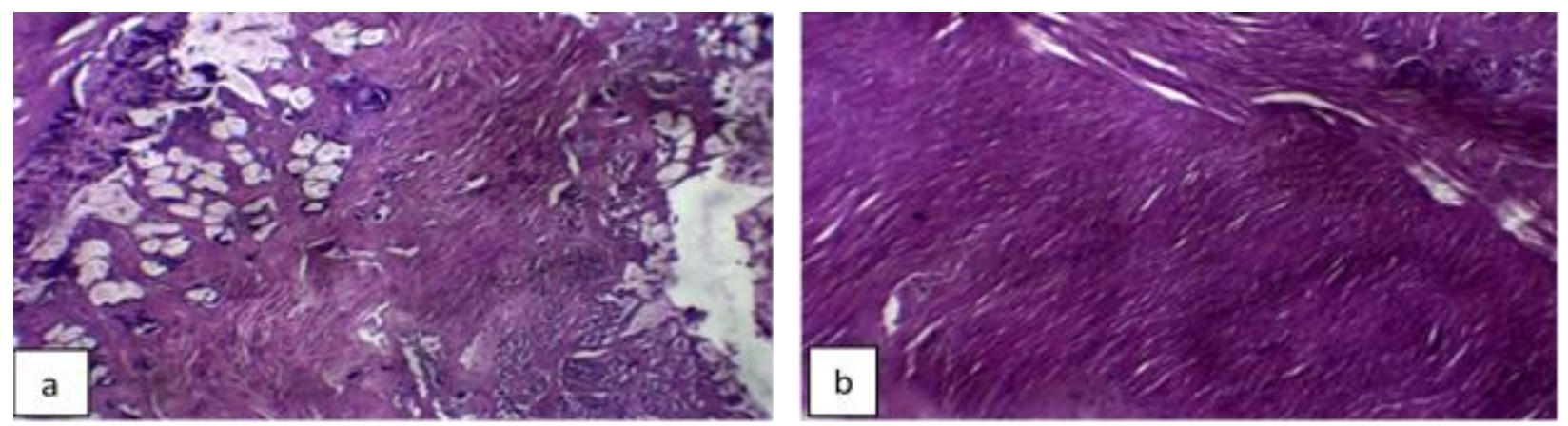

Figure 1. Histopathology of kidney tissue of frog stained with hematoxylin and eosin (H \& E) following CYP exposure a) Treated group kidney showing impairment of kidney tissue (b) Control group kidney.

Likewise, histological changes were evaluated in spleen of frog after exposure to alpha-cypermethrin showing disruption of centriole and abundance of white pulp part (Fig. 2). Histopathological sections of spleen

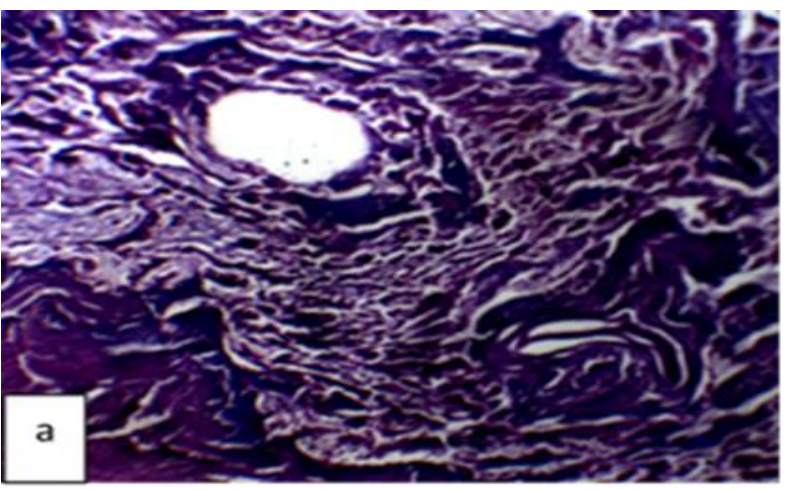

tissue exhibiting severe congestion are suggestive of alpha-cypermethin induced spleen damage (Fig. 2a) in contrast to control group showing normal histological patterns (Fig. 2b).

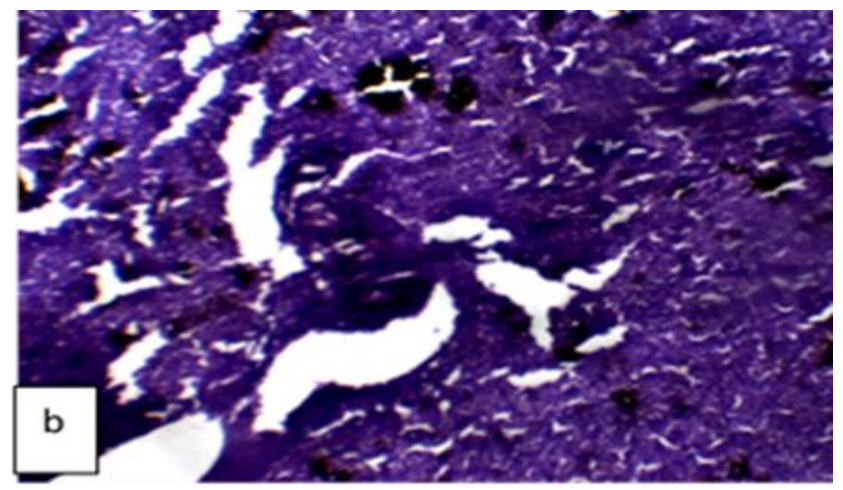

Figure 2. Histopathology of spleen tissue of frog stained with hematoxylin and eosin (H \& E) following CYP exposure a) Treated group spleen showing disruption of centriole (b) Control group spleen.

In the present study, the CYP mediated histopathological alterations in heart of frog were also studied (Fig. 3). Histology study of myocardial tissue carried out employing a light microscope and to investigate the effects of CYP, heart tissue was stained with $\mathrm{H}$ and $\mathrm{E}$ staining. Histopathological impact of alpha-Cypermethrin on heart tissue of treated frog was observed. CYP exposure caused heart injury in CYP exposed group. Photomicrography of frog heart after exposure to CYP showed intercalated disk and distorted cardiac muscle fibers present in treated frog heart (Fig. 3a) while normalized myocardial tissue structure of control heart tissue can be seen in (Fig. 3b). The controlgroup displayed no remarkable changes in 
vessels like no congestion while distinct myofibrils arrangement was noticed.

The toxic effects of CYP observed on histology of tongue tissue of treated group (Fig. 4). Photomicrography of frog tongue tissue reflected damaged distribution of tongue muscles and conical flame shaped cells were observed among treated group (Fig. 4a). The H \& E staining of untreated control group exhibited normal histology of tongue (Fig. 4b).
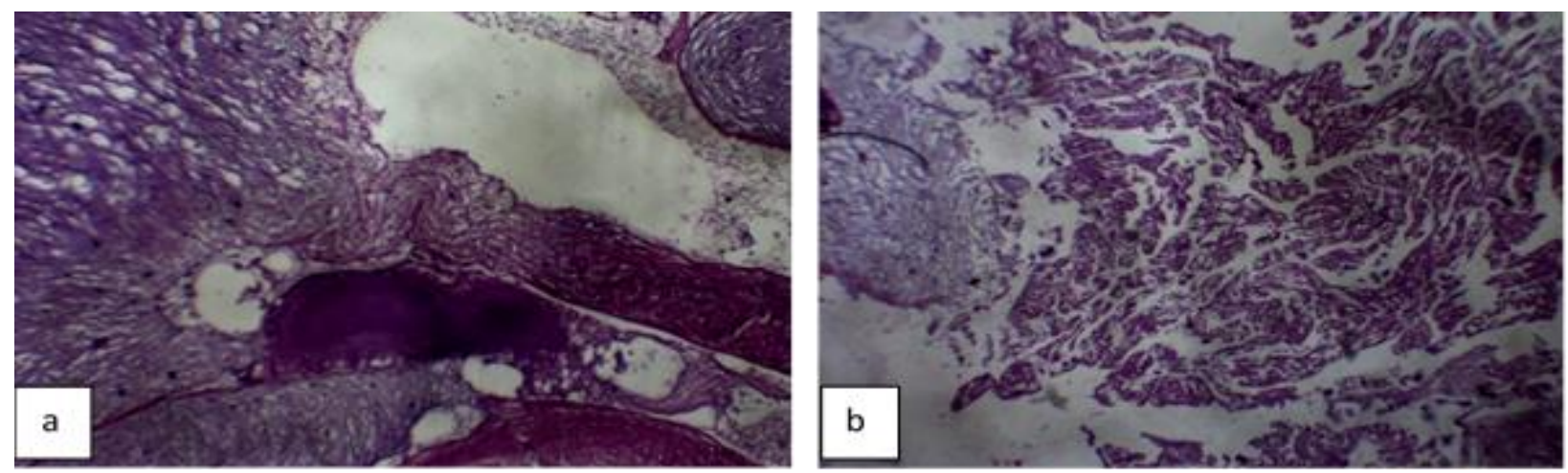

Figure 3. Histopathology of heart tissue of frog stained with hematoxylin and eosin (H \& E) following CYP exposure a) Treated frog heart showing intercalated disk and distorted cardiac muscles (b) Control group heart
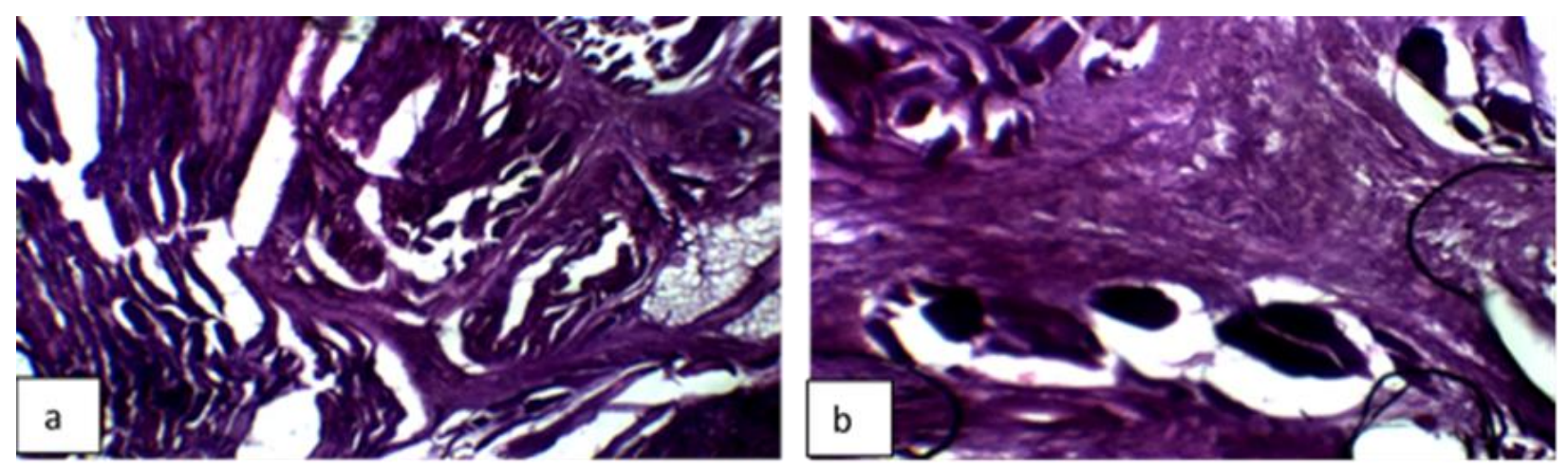

Figure 4. Histopathology of tongue tissue of frog stained with hematoxylin and eosin ( $\mathrm{H} \&$ E) following CYP exposure a) Micrograph of treated frog tongue showing damaged distribution of tongue muscles (b) Control group tongue tissue 


\section{Discussion}

Alpha-Cypermethrin (CYP) is a synthetic pyrethroid used as insecticide for large-scale effective control of dengue vectors. Studies have previously reported CYP to be extremely toxic to fish, bees and aquatic insects with possible death [11].

Over the past few decades' amphibian population decline has been observed worldwide with dozens of species vanishing due to several secondary factors including habitat destruction and modification, over population, and excessive use of pesticides [4]. In current study toxic effects of alphacypermethrin were evaluated by studying morphological changes in organ histology of adult male frog. Histopathological effects on kidney, heart, spleen, and tongue tissues of frog observed in consistence with Ghazouani, [12] showing exposure of alphacypermethrin resulting in cardiac injury and fibrosis. Disruptions in cardiac tissues histology were also reported in the current study showing cardiotoxicity which could effectively be caused by $\alpha$ - cypermethrin primarily due to oxidative stress and apoptosis.

The administration of cypermethrin caused significant changes in histology of kidney tissue. Studies reported that $\alpha$-cypermethrin affected the level of serum protein, and parameters of kidney profiles causing oxidative stress in animals [13].

Study has shown that differential sensitivity to cypermethrin assessed in amphibian larvae, varied with the stage at which exposure occurred, identified for $P$. regilla embryos. In early and later larval stages, cypermethrin was observed to induce mortality [14].

In studies conducted by Yassin and Hadi, [15] dose-dependent histological damages indicated the possible toxicity of cypermethrin in amphibians. Frog exposed to acute sub-lethal concentration of alphacypermethrin exhibited morphological changes in histology of treated frog's spleen assessed with signs of white pulp in treated group. While histological examination of tongue of treated group showed conical flame shaped cells and damaged distribution of tongue muscles showing that alphacypermethrin did induce slight morphological changes in the treatment group tongue tissues. Studies reported the toxic effects of cypermethrin pesticides has also been evaluated in some other studies [16].

The presence of amphibians can mask a lot of problems that can be manifested in long term consequences [17]. $\alpha$-Cypermethrin (CYP) a pyrethroid insecticide-like environmental pollutant, high levels exposure can link to health damage in frog.

\section{Conclusion}

Repeatedly deposition of anthropogenic chemicals may lead to associated unwanted toxicity to the non-targeted organisms in water bodies. The exposure of frog species to organophosphorus chemicals including alpha-cypermethin for an extended period of time may results in alteration of normal organ histology in them. Such pathological alterations in normal histological functions are reflecting that organophosphorus alphacypermethin at low concentrations cause pathology in frogs which can be used as bio indicator of aquatic ecosystem for such chemicals.

\section{Authors' contributions}

Conceived and designed the experiments: A Riaz, Performed the experiments: M Majeed, Analyzed the data: MA Riaz \& A Iqbal, Contributed materials/ analysis/tools: A Riaz \& UA Ashfaq, Wrote the paper: A Riaz \& M Majeed.

\section{References}

1. Bhunya SP \& Pati PC (1988). Genotoxic effects of a synthetic pyrethroid 
insecticide, cypermethrin, in mice in vivo. Toxicol Lett 41(3): 223-230.

2. Jin $Y$, Zheng S, Pu Y, Shu L, Sun L, Liu W \& Fu Z (2011). Cypermethrin has the potential to induce hepatic oxidative stress, DNA damage and apoptosis in adult zebrafish (Danio rerio). Chemosphere 82(3): 398-404.

3. Svartz G \& Coll CSP (2013). Comparative toxicity of cypermethrin and a commercial formulation on Rhinella arenarum larval development (Anura: Bufonidae). Int J Environ Health 6(4): 320-329.

4. Abdou HM, Hussien HM \& Yousef MI (2012). Deleterious effects of cypermethrin on rat liver and kidney: protective role of sesame oil. $J$ Environ Sci and Health B 47(4): 306-314.

5. Yousef MI, El-Demerdash FM \& AlSalhen KS (2003). Protective role of isoflavones against the toxic effect of cypermethrin on semen quality and testosterone levels of rabbits. J Environ Sci Health B 38(4): 463-478.

6. Sparling DW, Fellers GM \& McConnell LL (2001). Pesticides and amphibian population declines in California, USA. Environ Toxicol Chem 20(7): 1591-1595.

7. Salibian A \& Marazzo JL (1995). Studies on the effects of Deltamethrin on sodium net transport through the in vivo amphibian skin. Biomed Environ Sci 8(2): 164-168.

8. Afolabi OK, Aderibigbe FA, Folarin DT, Arinola A \& Wusu AD (2019). Oxidative stress and inflammation following sublethal oral exposure of cypermethrin in rats: mitigating potential of epicatechin. Heliyon 5(8): e02274.

9. Sushma N \& Devasena $T$ (2010). Aqueous extract of Trigonella foenum graecum (fenugreek) prevents cypermethrin-induced hepatotoxicity and nephrotoxicity. Hum Exp Toxicol 29(4): 311-319.
10. Bell RR, Nonavinakere VK \& Soliman MRI (2000). Intratracheal exposure of the Guinea Pig Lung to Cadmium and/or Selenium: A Histological Evaluation. Toxicol Lett 114: 101-109.

11. Singh AK, Tiwari MN, Upadhyay G, Patel DK, Singh D, Prakash O \& Singh MP (2010). Long term exposure to cypermethrin induces nigrostriatal dopaminergic neurodegeneration in adult rats: postnatal exposure enhances the susceptibility during adulthood. Neurobiol Aging 33(2): 404415.

12. Ghazouani L, Feriani A, Mufti A, Tir M, Baaziz I, Mansour HB \& Mnafgui K (2020). Toxic effect of alpha cypermethrin, an environmental pollutant, on myocardial tissue in male wistar rats. Environ Sci Pollut Resch 27: 5709-5717.

13. Soliman MM, Attia HF \& Abou El-Ella GA (2015). Genetic and histopathological alterations induced by cypermethrin in rat kidney and liver: protection by sesame oil. Int $J$ Immunopathol and Pharmacol 28(4): 508-520.

14. Biga LM \& Blaustein AR (2013). Variations in lethal and sublethal effects of cypermethrin among aquatic stages and species of anuran amphibians. Environ Toxicol Chem 32(12): 28552860.

15. Yassin FH \& Hadi AA (2016). Histopathological Alterations in Liver, Kidneys and Lungs Induced by Cypermethrin Toxicity in Albino Rats. $A l$ Kufa Uni J Biol 8(3): 275-285.

16. Ullah S, Zuberi A, Alagawany M, Farag MR, Dadar M, Karthik K, Tiwari R, Dhama K \& Iqbal HMN (2018). Cypermethrin induced toxicities in fish and adverse health outcomes: Its prevention and control measure 
adaptation. J Environ Manage 206: 863871.

17. Smalling KL, Reeves R, Muths E, Vandever M, Battaglin WA, Hladik ML
\& Pierce CL (2015). Pesticide concentrations in frog tissue and wetland habitats in a landscape dominated by agriculture. Sci Total Environ 502: 80-90. 